ISSN: $1130-3743$

DOI: http://dx.doi.org/10.14201/teoredu2014261137159

\title{
PRESENCIA DE LA COMPETENCIA MEDIÁTICA EN LOS OBJETIVOS CURRICULARES DE LA ETAPA DE EDUCACIÓN PRIMARIA
}

\author{
Presence of media competence in curricular objectives \\ of primary education
}

\section{Présence de la concurrence des médias dans les objectifs du programme d'études du cycle primaire}

Antonia Ramírez García*, Paula Renés Arellano** y María Rosa García Ruiz**

* Facultad de Ciencias de la Educación. Universidad de Córdoba.

Avda. San Alberto Magno, s/n. 14071 Córdoba. Correo-e: ed1ragaa@uco.es

* Universidad de Cantabria. Departamento de Educación. Avda. de Los Castros, s/n.39005 Santander.Correo-e: renesp@unican.es; rosa.garcia@unican.es

Fecha de recepción: enero de 2014

Fecha de aceptación: abril de 2014

Biblid [(1130-3743) 26, 1-2014, 137-159]

\section{RESUMEN}

Desde hace décadas los medios de comunicación han acaparado el interés de numerosos estudios. Sin embargo, los procesos de globalización y generalización de las tecnologías de la información y la comunicación, y la vinculación de estas últimas con los mass media, han provocado que este interés se acreciente entre diferentes instituciones internacionales. La educación no permanece ajena a esta temática, por el contrario, se encuentra preocupada por la influencia de los medios de comunicación en los procesos de enseñanza y aprendizaje del alumnado. Pero ¿hasta qué punto el currículo recoge esta preocupación? En este artículo analizamos la presencia de la competencia mediática, según las dimensiones establecidas 
por Ferrés (2007), en los objetivos de etapa y área de las comunidades autónomas de forma global. Los resultados muestran la presencia de esta competencia en las intenciones educativas, pero es necesario sistematizar y visibilizar la relación entre la educación y los medios.

Palabras clave: competencia mediática, currículo, educación primaria, medios de comunicación.

\section{SUMMARY}

For decades the mass media have centred the interest of numerous studies. Nevertheless, the processes of globalization and generalization of the technologies of the information and the communication, and the relation of the ITC with the mass comes up, have provoked that this interest increases between different international institutions. The education does not remain foreign to this subject-matter, on the contrary, it is worried for the influence of the mass media in the processes of education and learning of the pupils. But: until point the curriculum shows this worry? In this article we analyze the presence of the media competence, according to the dimensions established by Ferrés (2007), in the objectives of stage and area of the autonomous regions of global form. The results show the presence of this competence in the educational intentions, but it is necessary to systematize and to show explicitly the relation between the education and the mass media.

Key words: media literacy, curriculum, primary education, media.

\section{SOMMAIRE}

Depuis des décennies les médias ont accaparé l'intérêt de nombreuses études. Cependant, les processus de globalisation et de généralisation des technologies de l'information et la communication, et la relation du ITC avec les médias sert d'intermédiaire, a provoqué que cet intérêt s'accroît entre différentes institutions internationales. L'éducation ne reste pas étrangère à ce thème, au contraire, elle se trouve préoccupée par l'influence des médias sur les processus d'enseignement et l'apprentissage de l'ensemble des élèves. Cependant: jusqu'à ce qu'un point le curriculum scolaire recueille-t-il ce souci ? Dans cet article nous analysons la présence de la concurrence mediática, selon les dimensions établies par Ferrés (2007), dans les objectifs d'étape et d'aire des communautés autonomes de forme globale. Les résultats montrent la présence de cette concurrence dans les intentions éducatives, mais c'est un sistematizar nécessaire et montrer explicitement la relation entre l'éducation et les médias.

Mots clés: concurrence medias, curriculum, instruction primaire, médias. 


\section{INTRODUCCIÓN}

El interés de distintas comunidades autónomas por medir el grado de competencia mediática de la ciudadanía ha propiciado que, desde 2005 y hasta la actualidad, hayan proliferado los estudios realizados en torno a los medios de comunicación. El origen se encuentra en la investigación financiada por el Consell de l'Audiovisual de Catalunya (CAC) y por el Ministerio de Educación (Ferrés et al., 2011), en la que participaron 17 universidades pertenecientes a cada una de las comunidades autónomas y fue coordinada por la Universidad Pompeu Fabra de Barcelona. Esta investigación comenzó con la elaboración de un documento consensuado por un grupo de expertos en el que se definían las dimensiones y los indicadores que identificaban los límites de la competencia mediática (Ferrés, 2007). En la actualidad esta investigación se está complementando mediante la puesta en marcha de tres proyectos coordinados I+D+i, financiados por el Ministerio de Ciencia e Innovación y dirigidos respectivamente por Joan Ferrés, de la Universidad Pompeu Fabra; Ignacio Aguaded, de la Universidad de Huelva, y Agustín García Matilla, de la Universidad de Valladolid, Campus de Segovia. A través de estos tres proyectos se pretende realizar un diagnóstico de las necesidades que se detectan en tres grandes ámbitos vinculados con la competencia mediática de la ciudadanía: la enseñanza universitaria (en los ámbitos de la comunicación y de la educación), la enseñanza obligatoria y el colectivo de los profesionales de la comunicación.

En este artículo nos centraremos en el ámbito de la enseñanza obligatoria, concretamente en la etapa de educación primaria. En este sentido, hemos de partir de una realidad y ésta no es otra que de forma tradicional la educación, la escuela y los medios de comunicación han vivido una relación de continuos desencuentros, miedos, inseguridades, etc., que ha condicionado el camino seguido por cada uno. En el centro de todo ello se encuentra el alumno o alumna que vive en una sociedad denominada "del conocimiento", "de la información y comunicación" y que adquiere un conjunto de "conocimientos" en los centros educativos enmarcados en un proceso planificado de enseñanza y aprendizaje.

La incorporación de las competencias básicas al currículo escolar de la etapa de educación primaria posibilita el encuentro de ambos contextos formativos, pero su interconexión ha de responder a una intencionalidad, a un proceso de toma de decisiones reflexivo que permita integrar los diferentes conocimientos y situaciones comunicativas. En este sentido, el análisis de los elementos del currículo es fundamental para poder movilizar las dimensiones de cualquier competencia y hacer efectivos los aprendizajes en el aula.

\section{APROXIMACIÓN AL CURRÍCULO ESCOLAR DESDE UNA ÓPTICA COMPETENCIAL}

El debate en torno al currículo escolar no resulta algo novedoso; sin embargo, hasta él han llegado cuestiones como la calidad, la eficacia, los resultados, entre otros, que lo hacen convertirse en foco de interés para distintas autorías. 
En este sentido, la necesidad de una educación de calidad ha de "conectar el mundo escolar con las necesidades de la sociedad del siglo XXI, lo que exige replantear y reconstruir nuestro sistema educativo" (Arnaiz y De Haro, 2004, 21). De este modo, desde el Ministerio de Educación (2004) se elaboró el documento Una educación de calidad para todos y entre todos como marco para el debate de la nueva reforma educativa, que dos años después vería la luz de la mano de la Ley Orgánica 2/2006, de 3 de mayo, de Educación, y que pretendía servir para generar respuestas acordes a dichas necesidades.

Esta reforma no constituye algo aislado y exclusivo de España, desde finales del siglo xx se aprecia en el ámbito internacional una importante corriente de reformas que afecta de forma directa al currículum de los diferentes sistemas educativos. Braslawski $(2001,13)$ ha identificado cinco aspectos comunes a estas reformas educativas:

a) Consideración de la flexibilidad estructural.

b) Orientación de la formación hacia el desarrollo de competencias.

c) Tentativa de reducir la fragmentación por medio del estímulo de prácticas pedagógicas inter- y multidisciplinarias.

d) Introducción de opciones para los alumnos, cuando éstas no existan.

e) Recuperación de la pedagogía por proyectos.

En el caso de la reforma acometida en 2006, el currículo ha sido el elemento que mayor cambio ha experimentado. En este sentido, desde hace más de cuatro décadas han evolucionado diferentes teorías y definiciones del término currículo, tantas como autores. Estas teorías sobre el currículo representan distintas concepciones y diversas posibilidades para el análisis y formulación de propuestas, tanto teóricas, como prácticas. Algunas de ellas han basado el currículo en una planificación marcada por objetivos, nos referimos a las teorías racionalistas, entre las que destacan aquellas que promueven un currículum experiencial (Escuela Nueva y Dewey), las que giran en torno a un currículo normativo (Tyler y Taba) o tecnicista (Bloom y Gagné). Junto a ellas surgirán nuevas teorías como la reconceptualista (Huebner, Jackson o Pinar), el enfoque práctico (Schwab), el enfoque procesual (Stenhouse), el enfoque crítico (Apple) o el postestructuralismo de Silva o Moreira.

En lo que concierne a la definición de currículum, Taba (1974) lo entendía como un plan para el aprendizaje; Johnson (1982) como la suma de experiencias que los alumnos realizan mientras trabajan bajo la supervisión de la escuela, y para Román y Díez (citados por Tobón, 2006), es una selección cultural integrada por procesos (capacidades y valores), contenidos y métodos y procedimientos -formas de hacer- que demanda la sociedad en un momento determinado. Finalmente, el currículo, para Arroyo (2009), se configura como la acción estratégica de intervención que determina el éxito de las organizaciones educativas en su misión de ejecutar y concretar la visión de ser social y sociedad que tienen, vinculándolo, por tanto, al concepto de eficacia y a los modelos de gestión y evaluación. 
Así, pues, junto a la adopción de una determinada acepción del término currículo, los distintos países, desde finales del siglo xx, han tratado de buscar y encontrar fórmulas de diseño curricular que puedan conciliar las demandas e intereses de cada comunidad educativa con los más generales de sus respectivos contextos locales, regionales-autonómicos y nacionales.

La LOE (2006) en su capítulo III, artículo 6, entiende el currículo como "el conjunto de objetivos, competencias básicas, contenidos, métodos pedagógicos y criterios de evaluación de cada una de las enseñanzas reguladas". Esta definición supone la inclusión de un nuevo elemento curricular en el sistema educativo, las competencias básicas. Sin embargo, este elemento ha sido incorporado sin establecer una imbricación real con los demás elementos, produciéndose una situación de yuxtaposición curricular palpable. La necesidad de dar respuesta a las demandas europeas propició que se hiciera una adaptación de las denominadas por la Comisión Europea key competences y constituyeran parte del engranaje curricular. Su articulación en los diferentes Estados miembros ha dependido de la perspectiva educativa de cada país.

En este sentido, conviene recordar que el conocimiento ha ido evolucionando y transformándose a lo largo del tiempo de acuerdo con diferentes factores, lo que ha desembocado en distintas maneras de organizar los sistemas de formación y enseñanza. Así, De Ketele (2008) distingue cuatro grandes movimientos que categorizan a los sistemas educativos y los ha denominado con frases claramente definitorias: conocer es tener conocimiento de los textos clásicos y comentarlos, conocer es asimilar los resultados de los descubrimientos científicos y tecnológicos, conocer es demostrar dominio de objetivos traducidos en comportamientos observables y conocer es demostrar su competencia.

En este último movimiento es en el que nos situamos en la actualidad, tal y como apuntan todos los datos anteriores. Su origen se localiza, según De la Orden (2011, 47), en «el intento americano de cambiar en profundidad la capacitación inicial del profesorado de Educación Primaria (década de 1970) y la reforma del entrenamiento laboral y técnico-profesional en Europa, usA, Canadá y Australia (década de 1990)", pero poco a poco organismos internacionales como la OCDE, la UNESCO (http://www.unesco.org/new/en/education/themes/strengtheningeducation-systems/quality-framework/desired-outcomes/competencies/) o UNICEF (http://www.unicef.org/about/employ/files/UNICEF_Competencies.pdf) mostraban una preocupación por la insuficiencia del rendimiento educativo visto desde una óptica cuantitativa, por lo que se hacía necesario ampliar la visión hacia aspectos cualitativos. En este contexto se genera la idea de diseñar un currículo basado en el aprendizaje de competencias.

El término competencia ha sido definido desde diferentes ópticas, lo que le ha conferido un carácter polisémico. Algunas de estas definiciones han sido las ofrecidas por Escamilla (2009), Le Boterf (2000), Perrenoud (2004), Zabala y Arnau (2008) o el propio Proyecto DeSeCo (OCDE, 2002). Para Tardif $(2008,3)$, por ejemplo, se entiende como «un saber actuar complejo que se apoya sobre la 
movilización y la utilización eficaz de una variedad de recursos». Por su parte, Goñi $(2009,36)$ la define como el uuso eficiente y responsable del conocimiento para hacer frente a situaciones problemáticas relevantes". En ambos casos la competencia no supone un algoritmo memorizado y practicado de manera reiterada con el objetivo de que se almacene eternamente y pueda ser recuperado en el momento necesario, sino un saber actuar de carácter flexible y adaptado a distintos contextos y problemáticas.

La incorporación del "contexto" a la definición de competencia implica para Jonnaert, Barrette, Masciotra y Yaya (2008) la "cognición situada", es decir, una cognición vinculada a la práctica social y en ella los medios de comunicación juegan un papel destacado.

\section{LA IRRUPCIÓN DE LA COMPETENCIA MEDIÁTICA EN LA SOCIEDAD Y EN EL CURRÍCULO}

Como consecuencia del imparable desarrollo de las tecnologías de la información y la comunicación, de la omnipresencia de los medios de comunicación en nuestras vidas y de la preocupación de los poderes públicos por la audiencia de los mismos, el término competencia mediática, competencia en medios de comunicación, competencia en comunicación o competencia audiovisual, como se la ha denominado en diferentes contextos, se ha convertido en centro de interés por parte de distintos investigadores como Bazalgette, Bevort, Buckingham, Carlsson, Piette, Tisseron o Verniers (Aguaded, 2011, 19-20) apuntaron, quienes han abogado por que se convierta en una competencia básica adquirida por toda la ciudadanía.

Esta vinculación entre la sociedad, los medios de comunicación y la educación ha propiciado que Coll y Martín (2006) hayan realizado una serie de reflexiones, destacando dos de todas ellas. En primer lugar la que alude a la funcionalidad de las competencias como movilizadoras de saberes y facilitadoras de la aplicación de los mismos en diferentes contextos. En segundo lugar, la utilización del concepto alfabetización (en inglés literacy) para hacer referencia a una cultura determinada (matemática, letrada, científica, tecnológica, visual, entre otras), a las herramientas simbólicas que emplea (lenguaje matemático, lengua escrita, lenguaje científico, lenguaje tecnológico, visual, etc.), al desarrollo de prácticas socioculturales concretas (leer el periódico para informarse, leer textos científicos para formarse, leer poesía para disfrutar, etc.) y a los saberes asociados a dichas prácticas (qué es un periódico, dónde se puede encontrar, cómo se organiza, cómo se valora la fiabilidad de la información que ofrece, etc.). Por su parte, Medrano (2008, 208209) apunta más allá al afirmar que tanto los fenómenos mediáticos como las preferencias de consumo de las audiencias "se pueden considerar como indicadores de un cierto estado cultural" de las mismas, esto supone iniciar "un círculo vicioso" de formación y reproducción "de valores, normas y patrones de consumo mediáticocultural" por lo que es necesario una pedagogía de los medios. En la actualidad, esta pedagogía encuentra una posibilidad de desarrollo en el ámbito educativo. 
En este sentido, Coll y Martín (2006, 9-10) proponen definir unos aprendizajes básicos, en este caso pueden estar marcados por los objetivos mínimos establecidos en la normativa actual tanto a nivel de etapa como de áreas curriculares, y proporcionar al alumnado una serie de "herramientas simbólicas, prácticas socioculturales y saberes".

Estos objetivos han quedado establecidos en la LOE (2006) y en el Real Decreto $1513 / 06$, de 7 de diciembre, mientras que estas herramientas y prácticas forman parte de los descriptores de las seis dimensiones que, a juicio de Ferrés (2007), integran la competencia mediática o audiovisual.

En cuanto a los objetivos, estos han sido definidos por Bloom (1956) como un fin o resultado esperado en las experiencias educativas, para Ausubel (1976) es lo que un alumno debe poder hacer o decir cuando ha terminado la educación. Los objetivos educativos además muestran las orientaciones del modelo de formación que se adopta, el conductista o el cognitivista, entre otros, y que es el propuesto por el legislador. En nuestro sistema educativo, concretamente en la etapa de educación primaria, las metas educativas se han categorizado en objetivos de etapa y de área, expresados en términos de capacidades. Estos son los que en párrafos posteriores vamos a analizar en relación con la competencia mediática.

En lo que concierne a la competencia que estamos abordando en estas páginas, Ferrés $(2007,10)$ ha definido la competencia audiovisual como:

La capacidad de un individuo para interpretar y analizar desde la reflexión crítica las imágenes y los mensajes audiovisuales, y para expresarse con una mínima corrección en el ámbito comunicativo. Esta competencia está relacionada con el conocimiento de los medios de comunicación y con el uso básico de las tecnologías multimedia necesarias para producirla.

Por su parte, González, Sedeño y Gonzálvez (2012, 122) consideran que «la competencia audiovisual está íntimamente relacionada con la competencia mediática, de modo que pueden incluso identificarse ambas categorías en un entorno en el que es fundamental el conocimiento de los medios de comunicación y de las tecnologías multimedia". En esta misma dirección, Ferrés y Piscitelli $(2012,79)$ han precisado que:

La competencia mediática comporta el dominio de conocimientos, destrezas y actitudes relacionados con seis dimensiones básicas, de las que se ofrecen los indicadores principales. Estos indicadores tienen que ver, según los casos, con el ámbito de participación como personas que reciben mensajes e interaccionan con ellos (ámbito del análisis) y como personas que producen mensajes (ámbito de la expresión).

Estas dimensiones básicas de la competencia mediática aparecen definidas en la Figura 1. 
Figura 1. DEFINICIÓN DE LAS SEIS DIMENSIONES DE LA COMPETENCIA MEDiÁTICA

\begin{tabular}{|c|c|}
\hline DiMENSIÓN & DEFINICIÓN \\
\hline Lenguaje & $\begin{array}{l}\text { Conocimiento de los códigos que hacen posible el lenguaje visual y capacidad } \\
\text { de utilizarlos para comunicarse de manera sencilla pero efectiva. } \\
\text { Capacidad de análisis de los mensajes audiovisuales desde la perspectiva } \\
\text { del sentido y la significación, de las estructuras narrativas y de las categorías } \\
\text { y géneros. }\end{array}$ \\
\hline Tecnología & $\begin{array}{l}\text { Conocimiento teórico del funcionamiento de las herramientas que hacen } \\
\text { posible la comunicación audiovisual, para poder entender cómo son } \\
\text { elaborados los mensajes. } \\
\text { Capacidad de utilización de las herramientas más sencillas para comunicarse } \\
\text { de manera eficaz en el ámbito de lo audiovisual. }\end{array}$ \\
\hline Procesos de interacción & $\begin{array}{l}\text { Capacidad de reconocerse como audiencia activa, especialmente a partir } \\
\text { del uso de las tecnologías digitales que permiten la participación y la } \\
\text { interactividad. } \\
\text { Capacidad de valorar críticamente los elementos emotivos, racionales y } \\
\text { contextuales que intervienen en la recepción y valoración de los mensajes } \\
\text { audiovisuales. }\end{array}$ \\
\hline $\begin{array}{c}\text { Procesos de } \\
\text { producción y difusión }\end{array}$ & $\begin{array}{l}\text { Conocimiento de las funciones y tareas asignadas a los principales agentes } \\
\text { de producción y las fases en las que se descomponen los procesos } \\
\text { de producción y programación de los distintos tipos de producciones } \\
\text { audiovisuales. } \\
\text { Capacidad de elaborar mensajes audiovisuales y conocimiento de su } \\
\text { trascendencia e implicaciones en los nuevos entornos de comunicación. }\end{array}$ \\
\hline Ideología y valores & $\begin{array}{l}\text { Capacidad de lectura comprensiva y crítica de los mensajes audiovisuales, en } \\
\text { cuanto representaciones de la realidad y, en consecuencia, como portadores } \\
\text { de ideología y valores. } \\
\text { Capacidad de análisis crítico de los mensajes, entendidos a un tiempo como } \\
\text { expresión y soporte de los intereses, de las contradicciones y de los valores } \\
\text { de la sociedad. }\end{array}$ \\
\hline Estética & $\begin{array}{l}\text { Capacidad de analizar y valorar los mensajes audiovisuales desde el punto } \\
\text { de vista de la innovación formal y temática y la educación del sentido } \\
\text { estético. } \\
\text { Capacidad de relacionar los mensajes audiovisuales con otras formas de } \\
\text { manifestación mediática y artística. }\end{array}$ \\
\hline
\end{tabular}

FuENTE: Elaboración propia a partir de Ferrés $(2007,103)$.

Para Ferrés (2007) estas dimensiones no constituyen compartimentos estancos en los que sus descriptores quedan anclados, todas ellas se relacionan y, en ocasiones, resulta difícil delimitar dónde finaliza una y comienza la siguiente.

\section{OBJETIVOS Y PLANTEAMIENTO METODOLÓGICO}

El punto de partida de esta investigación se sitúa en las seis dimensiones mencionadas con anterioridad -lenguajes, tecnología, procesos de interacción, procesos de producción y difusión, ideología y valores y estética- y sus 
correspondientes indicadores. A partir de ahí, se ha procedido a revisar el currículo escolar de todas las comunidades autónomas, recogido en la diferente normativa. El objetivo consistía en determinar la presencia o ausencia de la competencia mediática en el currículo de la etapa de Educación Primaria.

Como consecuencia de este objetivo, el interrogante de investigación propuesto parte de una serie de afirmaciones realizadas por distintos autores (Aparici, Campuzano, Ferrés y García, 2010; Camps, 2009 y Tucho, 2008) sobre la escasa presencia de la competencia mediática en el currículo de las etapas de enseñanza obligatoria, pero ¿cuál es la presencia real de la competencia mediática en los objetivos curriculares que el alumnado de educación primaria ha de alcanzar?

Esta revisión se ha realizado en el marco del Proyecto I+D La enseñanza obligatoria ante la competencia en comunicación audiovisual en un entorno digital (EDU2010-21395-CO3-03), como fase previa al diseño de un cuestionario destinado a medir la competencia mediática del alumnado de cuarto de educación primaria (ver cuestionario en http://www.uhu.es/competenciamediatica/primaria/).

El método que se ha empleado en esta investigación ha sido el análisis documental y el proceso seguido puede observarse de manera gráfica en la Figura 2.

\section{FIGURA 2. PROCESO DE VINCULACIÓN}

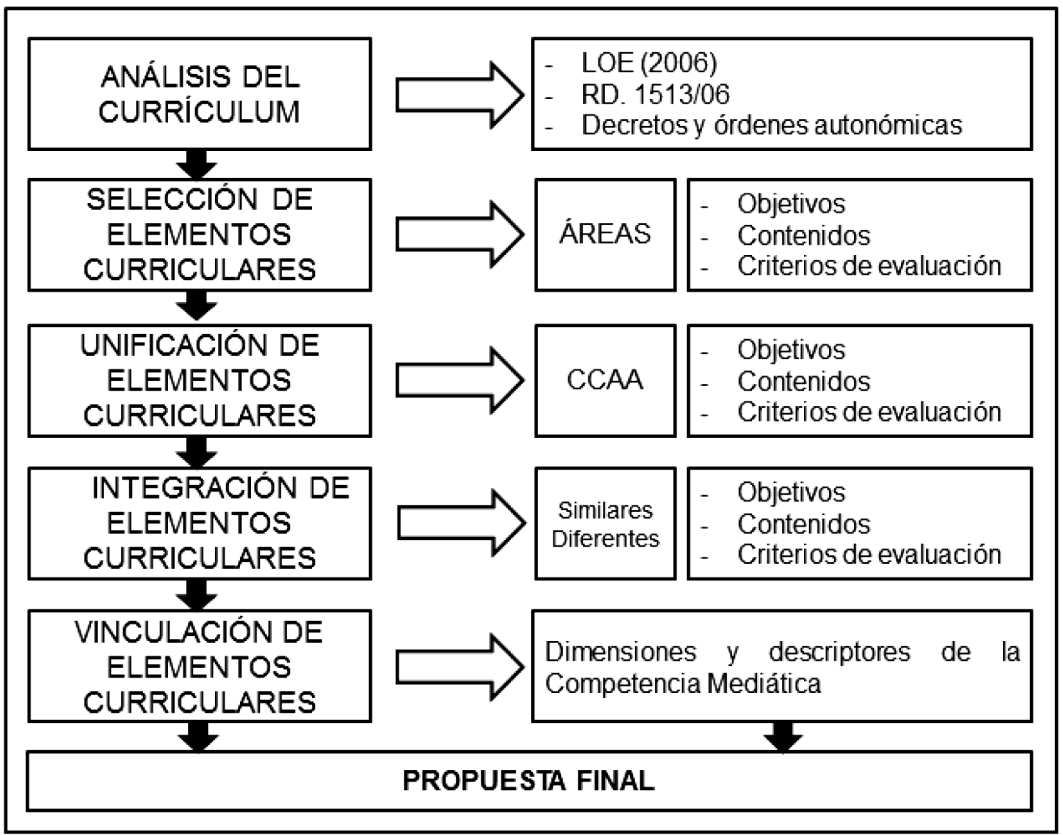

FuENTE: Elaboración propia. 
La rigurosidad y sistematicidad han caracterizado a cada una de estas fases, siendo la que mayor dificultad ha presentado la elaboración de una propuesta final en la que se relacionaban los descriptores de la competencia mediática con los objetivos del currículo de cuarto de educación primaria.

La elección de los objetivos como elemento de análisis estriba en que estos son las metas que guían todo el currículum y la práctica diaria en las aulas, ya que son el referente último al que se ha de aspirar. De este modo, los objetivos, al estar expresados en términos de capacidades, podrían reflejar la presencia o ausencia de la competencia mediática en el currículo escolar y en los centros educativos.

En las cuatro primeras fases han participado las autoras del artículo, especialistas en medios de comunicación y vinculadas de manera directa con el ámbito educativo. En la quinta fase, junto a las mismas, han intervenido diez maestros y maestras de educación primaria en ejercicio ${ }^{1}$, siendo su tarea establecer la vinculación entre los descriptores de la competencia mediática y los contenidos del currículum. La propuesta presentada en estas páginas ha tenido en consideración que la vinculación entre ambos elementos se produjera, al menos, en el 70\% de las respuestas dadas por los participantes.

\section{Resultados}

Si analizamos los estudios sobre la competencia mediática en el ámbito educativo, podemos destacar dos aspectos fundamentales que señalan: la escasez de los propios estudios y la reducida presencia de la competencia mediática en los currículos (Camps, 2009; Aparici, Campuzano, Ferrés y García, 2010 y Tucho, 2008). No obstante, esta última circunstancia es necesaria explicitarla con evidencias concretas, en este apartado intentaremos que así sea.

Los resultados que ofrecemos a continuación se van a presentar de acuerdo a los dos tipos de objetivos que se establecen en el currículo de la etapa de educación primaria: los objetivos de etapa y los objetivos de área.

Así, en primer lugar, uno de los objetivos de etapa que ha de alcanzar el alumnado en todas las comunidades autónomas es iniciarse en el uso de las tecnologías de la información y de la comunicación (TIC) con la finalidad de aprender y comunicarse, desarrollando un espíritu crítico ante los mensajes que reciben y elaboran. La comunidad autónoma de Cataluña añade en relación con este objetivo que el alumnado ha de saber seleccionar y valorar la información recibid a por las Tic. Por su parte, Asturias y Castilla-La Mancha incorporan que las Tic han de servir no sólo para el aprendizaje, sino también para la comunicación interpersonal. Asimismo,

1. Manuel J. Montilla, Cristina López, Ana M.a Granados, Diego Baena, Juana Sánchez, Antonio Saavedra, Carolina Moreno, M..$^{a}$ Dolores Montilla, Juan José López y Carlos A. García. 
Extremadura insiste en que estas han de utilizarse también para el desarrollo personal y social. Este objetivo se vincula, pues, con las siguientes tres dimensiones:

a) Dimensión Ideología y valores (ámbito del análisis): capacidad de detectar las intenciones o intereses que subyacen tanto en las producciones corporativas como en las populares, así como su ideología y valores, explícitos o latentes, adoptando una actitud crítica ante ellos.

b) Dimensión Lenguajes (ámbito del análisis): capacidad de analizar y de valorar los mensajes desde la perspectiva del significado y del sentido, de las estructuras narrativas y de las convenciones de género y de formato.

c) Dimensión Tecnología (ámbito del análisis): comprensión del papel que desempeñan en la sociedad las TIC y de sus posibles efectos.

El segundo objetivo vinculado a la competencia mediática alude a la capacidad de utilizar las representaciones y expresiones artísticas e iniciarse en la construcción de propuestas visuales. En Aragón se solicita además al alumnado que desarrolle la sensibilidad estética y la creatividad. Asturias, por su parte, incluye la capacidad para disfrutar de las obras y manifestaciones artísticas. Algunas comunidades autónomas como Castilla y León, Madrid, Murcia y Valencia van más allá y consideran que el alumnado ha de ser capaz de comunicarse a través de distintos medios de expresión verbal, corporal, visual, etc., y no solo utilizarlos como receptores. Por su parte, la comunidad de Islas Baleares incorpora en este objetivo la capacidad de valorar las manifestaciones artísticas para comunicarse en distintos lenguajes. Por último, el País Vasco realiza una síntesis de todas las aportaciones de las diferentes comunidades autónomas mencionadas.

En el caso de este segundo objetivo, las dimensiones lenguajes y estética son las que presentan una mayor relación con él, concretamente los siguientes descriptores:

a) Dimensión Lenguajes (ámbito de la expresión): capacidad de expresarse mediante una amplia gama de sistemas de representación y de significación.

b) Dimensión Estética (ámbitos del análisis y la expresión):

- Capacidad de extraer placer de los aspectos formales, es decir, no solo de lo que se comunica, sino también de la manera como se comunica.

- Capacidad de producir mensajes elementales que sean comprensibles y que contribuyan a incrementar los niveles personales o colectivos de creatividad, originalidad y sensibilidad.

En lo que concierne al segundo tipo de objetivos, de los 55 descriptores de la competencia mediática, 20 han quedado identificados en los objetivos de las áreas que integran el currículo de educación primaria: Conocimiento del medio, Educación artística, Educación física, Lengua castellana, Lengua extranjera, Matemáticas y Segunda lengua extranjera. Este número supone que el 36,36\% de los aspectos distintivos de la competencia mediática se encuentra presente en dicho currículo, 
pero cómo se encuentra distribuida dicha presencia. En la Figura 3 podemos apreciar la comparación entre la propuesta de Ferrés (2007) y los objetivos de las distintas áreas. Las mayores diferencias entre ambos se aprecian en tres dimensiones: procesos de interacción, procesos de producción y difusión e ideología y valores.

Figura 3. PRESENCIA de los objetivos de las ÁREAS CURRICUlares EN LOS DESCRIPTORES DE LA COMPETENCIA MEDIÁTICA

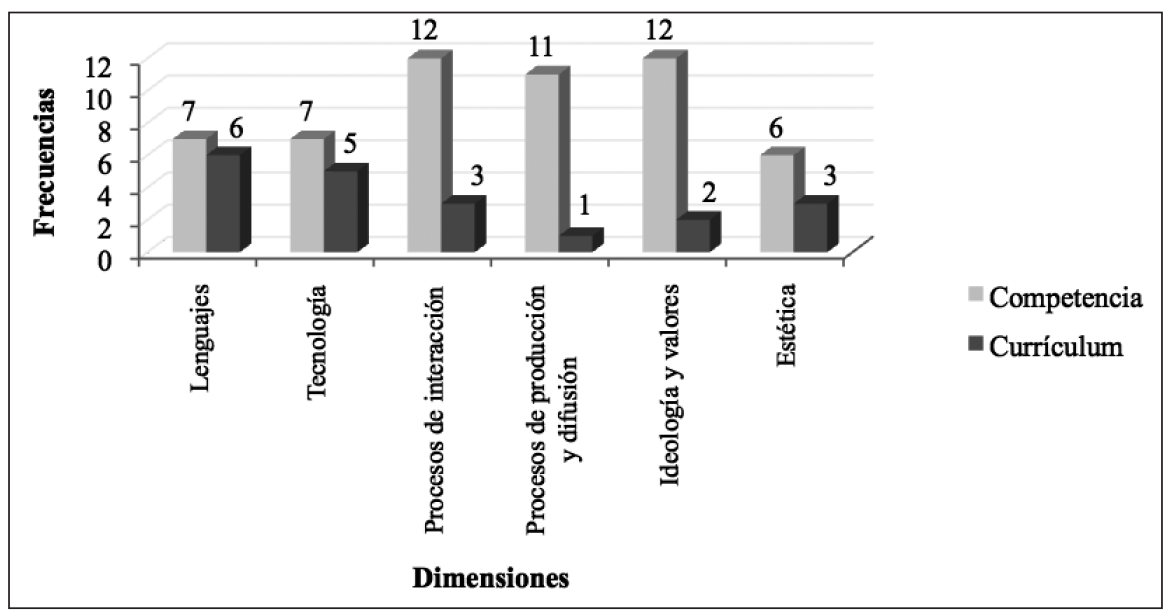

FUENTE: Elaboración propia.

Figura 4. Distribución de lOS OBJETIVOS DE LAS ÁREAS CURRICULARES EN FUNCIÓN DE LAS DIMENSIONES Y SUBDIMENSIONES DE LA COMPETENCIA DIGITAL

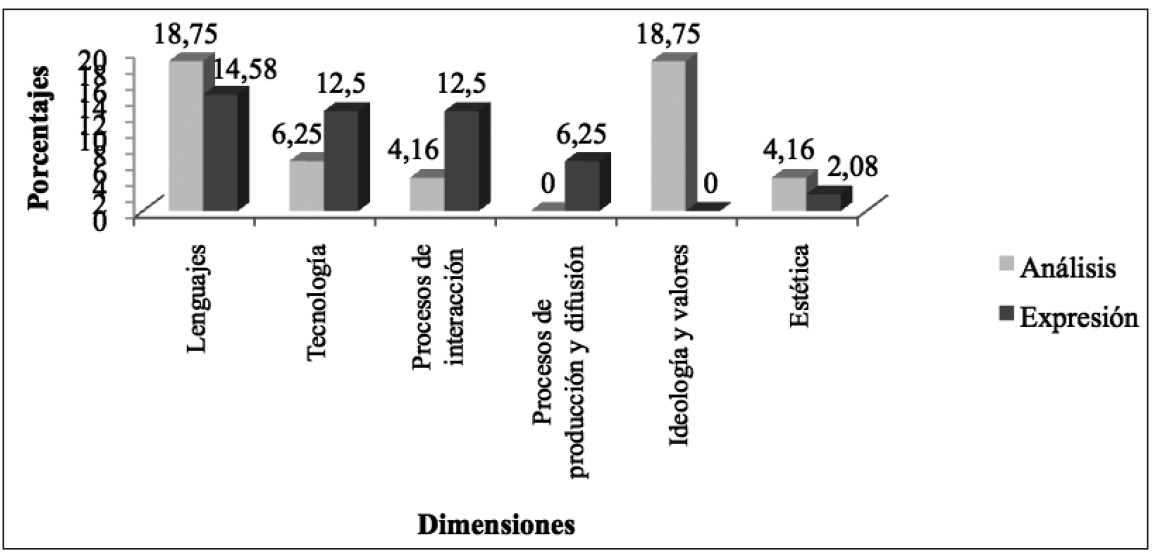

FUENTE: Elaboración propia. 
Por su parte, en la Figura 4 destacamos la distribución de esta presencia en los dos ámbitos que integran cada una de las dimensiones, el del análisis y el de la expresión. Estos dos ámbitos posibilitan que la persona reconozca su competencia mediática "interaccionando de manera crítica con los mensajes producidos por los demás, y siendo capaz de producir y de diseminar mensajes propios" (Ferrés y Psicitelli, 2012, 77-78). De la misma podemos extraer cuatro cuestiones básicas:

1. En las dimensiones lenguajes, ideología y valores y estética predominan los procesos de análisis frente a los de expresión.

2. En las dimensiones tecnología, procesos de interacción y procesos de producción y difusión sobresalen los procesos de expresión, frente a los de análisis.

3. En la dimensión procesos de producción y difusión solo se contemplan procesos de expresión.

4. En la dimensión ideología y valores solo se aprecia la presencia de los procesos de análisis.

De forma más detallada, en las Tablas 1 a 6 se muestran las frecuencias y los porcentajes de los objetivos de las áreas del currículo de educación primaria relacionados con los descriptores de la competencia mediática en cada una de sus dimensiones.

Las áreas de Educación artística (EA), Lengua extranjera (LE) y Lengua castellana (LC) son las que mayor presencia manifiestan en la dimensión Lenguajes, seguidas de Conocimiento del medio (CM). Tanto en Matemáticas (MA) como en Educación Física (EF) esta presencia es más reducida (Ver Tabla 1).

TABla 1. Frecuencias y PORCENTAJeS totales De los Descriptores DE LA DIMENSIÓN LENGUAJES

\begin{tabular}{|l|l|c|c|c|c|c|c|c|}
\hline \multicolumn{2}{|c|}{ DESCRIPTORES } & CM & EA & EF & LC & LE & MA & $\%$ \\
\hline \multicolumn{2}{|l|}{$\begin{array}{l}\text { L1a. Capacidad de interpretar y de valorar los } \\
\text { diversos códigos de representación y la función } \\
\text { que cumplen en un mensaje. }\end{array}$} & 1 & 1 & 0 & 0 & 0 & 0 & 4,16 \\
\hline $\begin{array}{l}\text { L2a. Capacidad de analizar y de valorar los } \\
\text { mensajes desde la perspectiva del significado y } \\
\text { del sentido, de las estructuras narrativas y de las } \\
\text { convenciones de género y de formato. }\end{array}$ & 0 & 1 & 0 & 0 & 0 & 0 & 2,08 \\
\hline $\begin{array}{l}\text { L3a. Capacidad de comprender el flujo de } \\
\text { historias y de informaciones procedentes de } \\
\text { múltiples medios, soportes, plataformas y } \\
\text { modos de expresión. }\end{array}$ & 0 & 0 & 0 & 2 & 3 & 0 & 10,41 \\
\hline $\begin{array}{l}\text { L4a. Capacidad de establecer relaciones } \\
\text { entre textos -intertextualidad-, códigos y } \\
\text { medios, elaborando conocimientos abiertos, } \\
\text { sistematizados e interrelacionados. }\end{array}$ & 1 & 0 & 0 & 0 & 0 & 0 & 2,08 \\
\hline
\end{tabular}




\begin{tabular}{|c|l|c|c|c|c|c|c|c|}
\hline \multicolumn{2}{|c|}{ DESCRIPTORES } & CM & EA & EF & LC & LE & MA & $\%$ \\
\hline \multirow{2}{*}{$\begin{array}{l}\text { L1e. Capacidad de expresarse mediante una } \\
\text { amplia gama de sistemas de representación y } \\
\text { de significación. }\end{array}$} & 0 & 2 & 1 & 1 & 0 & 0 & 8,33 \\
\cline { 2 - 10 } & $\begin{array}{l}\text { L2e. Capacidad de elegir entre distintos } \\
\text { sistemas de representación y distintos estilos } \\
\text { en función de la situación comunicativa, del } \\
\text { tipo de contenido que hay que transmitir y del } \\
\text { tipo de interlocutor. }\end{array}$ & 0 & 1 & 0 & 0 & 1 & 1 & 6,25 \\
\hline \multicolumn{1}{|c|}{ TOTAL } & 2 & 5 & 1 & 3 & 4 & 1 & 33,33 \\
\hline
\end{tabular}

FUENTE: Elaboración propia.

Algunos de los ejemplos que podemos citar son los siguientes:

Educación artística:

- Indagar en las posibilidades del sonido, la imagen y el movimiento como elementos de representación y comunicación y utilizarlas para expresar, valorar y aceptar hechos, ideas y sentimientos, contribuyendo con ello al equilibrio afectivo y a la ampliación de las capacidades relacionales y comunicativas (L1e).

Educación física:

- Comprender y expresar correctamente documentos y mensajes orales, escritos, corporales y audiovisuales propios del área (L1e).

Lengua castellana:

- Comprender discursos orales y escritos de diferentes ámbitos de uso e interpretarlos para aplicar la comprensión de los mismos a nuevas situaciones de comunicación y aprendizaje (L3a).

Lengua extranjera:

- Planificar, organizar y escribir textos diversos con finalidades variadas y estructura adecuada a los diferentes tipos de discurso, utilizando diferentes soportes y fuentes de información (L2e).

Por su parte, en la dimensión Tecnología, las áreas de Educación artística, Educación física y Lengua extranjera ocupan el primer lugar en cuanto a presencia de los descriptores de la competencia digital, seguidas de Conocimiento del medio, Lengua castellana y Matemáticas (ver Tabla 2). Los objetivos más significativos son los que se expresan seguidamente: 


\begin{tabular}{|c|c|c|c|c|c|c|c|c|}
\hline \multicolumn{2}{|r|}{ DESCRIPTORES } & $\mathrm{CM}$ & EA & $\mathrm{EF}$ & LC & LE & MA & $\%$ \\
\hline \multirow{3}{*}{ 总 } & $\begin{array}{l}\text { T1a. Comprensión del papel que } \\
\text { desempeñan en la sociedad las tecnologías } \\
\text { de la información y de la comunicación y } \\
\text { de sus posibles efectos. }\end{array}$ & 0 & 1 & 0 & 0 & 0 & 0 & 2,08 \\
\hline & $\begin{array}{l}\text { T2a. Habilidad para interactuar de manera } \\
\text { significativa con medios que permiten } \\
\text { expandir las capacidades mentales. }\end{array}$ & 0 & 0 & 0 & 0 & 0 & 1 & 2,08 \\
\hline & $\begin{array}{l}\text { T3a. Capacidad de desenvolverse con } \\
\text { eficacia en entornos hipermediales, } \\
\text { transmediáticos y multimodales. }\end{array}$ & 0 & 0 & 1 & 0 & 0 & 0 & 2,08 \\
\hline \multirow{2}{*}{ 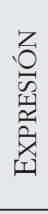 } & $\begin{array}{l}\text { T1e. Capacidad de manejar con corrección } \\
\text { herramientas comunicativas en un entorno } \\
\text { multimedial y multimodal. }\end{array}$ & 0 & 1 & 1 & 1 & 2 & 0 & 10,41 \\
\hline & $\begin{array}{l}\text { T2e. Capacidad de adecuar las herramientas } \\
\text { tecnológicas a los objetivos comunicativos } \\
\text { que se persiguen. }\end{array}$ & 1 & 0 & 0 & 0 & 0 & 0 & 2,08 \\
\hline \multicolumn{2}{|r|}{ TOTAL } & 1 & 2 & 2 & 1 & 2 & 1 & 18,75 \\
\hline
\end{tabular}

FUENTE: Elaboración propia.

Conocimiento del medio:

- Utilizar las TIC para obtener información y como instrumento para aprender y compartir conocimientos, manteniendo una actitud crítica ante su uso y sus contenidos, valorando su contribución a la mejora de las condiciones de vida de todas las personas (T1a).

Educación artística:

- Conocer y utilizar la lectura, los medios audiovisuales y las Tic como recurso artístico y como instrumento de aprendizaje (T1e).

Educación física:

- Utilizar las tecnologías de la información y la comunicación como recurso de apoyo al área y a la regulación de los aprendizajes (T3a).

Lengua extranjera y segunda lengua extranjera:

- Aprender a utilizar con progresiva autonomía todos los medios a su alcance, incluidas las tecnologías de la información y la comunicación, y para comunicarse en la lengua extranjera (T1e). 
Matemáticas:

- Utilizar el conocimiento matemático para comprender, valorar y producir informaciones y mensajes sobre hechos y situaciones de la vida cotidiana y reconocer su carácter instrumental para otros campos de conocimiento (T2e).

En cuanto a la dimensión Procesos de interacción, las áreas del lenguaje -Lengua castellana y Lengua extranjera- encabezan la mayor relación con sus descriptores, seguidas de Educación artística. Sin embargo, no existen evidencias de esta dimensión en las áreas de Conocimiento del medio, Educación física y Matemáticas (ver Tabla 3).

\section{TABla 3. FRECUENCIAS Y PORCENTAJES TOTALES DE LOS DESCRIPTORES DE LA DIMENSIÓN PROCESOS DE INTERACCIÓN}

\begin{tabular}{|c|c|c|c|c|c|c|c|c|}
\hline \multicolumn{2}{|r|}{ DESCRIPTORES } & $\mathrm{CM}$ & EA & $\mathrm{EF}$ & LC & LE & MA & $\%$ \\
\hline 窟 & $\begin{array}{l}\text { I3a. Capacidad de discernir y de gestionar } \\
\text { las disociaciones que se producen a } \\
\text { veces entre sensación y opinión, entre } \\
\text { emotividad y racionalidad. }\end{array}$ & 0 & 0 & 0 & 1 & 1 & 0 & 4,16 \\
\hline \multirow{2}{*}{ 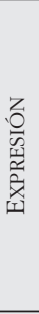 } & $\begin{array}{l}\text { I1e. Actitud activa en la interacción con las } \\
\text { pantallas, entendidas como oportunidad } \\
\text { para construir una ciudadanía más plena, } \\
\text { un desarrollo integral, para transformarse } \\
\text { y para transformar el entorno. }\end{array}$ & 0 & 2 & 0 & 1 & 1 & 0 & 8,33 \\
\hline & $\begin{array}{l}\text { I2e. Capacidad de interaccionar con } \\
\text { personas y con colectivos diversos } \\
\text { en entornos cada vez más plurales y } \\
\text { multiculturales. }\end{array}$ & 0 & 0 & 0 & 1 & 1 & 0 & 4,16 \\
\hline \multicolumn{2}{|r|}{ TOTAL } & 0 & 2 & 0 & 3 & 3 & 0 & 16,66 \\
\hline
\end{tabular}

FUENTE: Elaboración propia.

Como ejemplo, podemos mencionar los siguientes objetivos de las áreas curriculares:

Educación artística:

- Desarrollar una actitud crítica y selectiva ante la información de los medios de comunicación (I1e).

Lengua castellana y lengua extranjera:

- Valorar la realidad multilingüe y cultural de nuestra sociedad como fuente de riqueza personal y colectiva, tomar conciencia de la importancia del 
dominio de lenguas en un mundo cada vez más global y para utilizar con progresiva autonomía todos los medios a su alcance, incluyendo las TIC, para obtener información y para comunicarse, evitando los estereotipos lingüísticos que suponen juicios de valor y prejuicios clasistas, racistas o sexistas (I2e).

En lo que concierne a la dimensión Procesos de producción y difusión nuevamente las áreas lingüísticas, junto con Matemáticas, son las que mayor vinculación mantienen con la competencia mediática. El resto de las áreas, Conocimiento del medio, Educación artística y Educación física, no establecen ninguna vinculación con la misma (Ver Tabla 4).

\section{TABLA 4. FRECUENCIAS Y PORCENTAJES TOTALES DE LOS DESCRIPTORES DE LA DIMENSIÓN PROCESOS DE PRODUCCIÓN Y DIFUSIÓN}

\begin{tabular}{|c|c|c|c|c|c|c|c|c|}
\hline \multicolumn{2}{|r|}{ DESCRIPTORES } & $\mathrm{CM}$ & EA & $\mathrm{EF}$ & LC & $\mathrm{LE}$ & MA & $\%$ \\
\hline 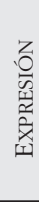 & $\begin{array}{l}\text { P4e. Capacidad de compartir y diseminar } \\
\text { información, a través de los medios } \\
\text { tradicionales y de las redes sociales, } \\
\text { incrementando la visibilidad de los } \\
\text { mensajes, en interacción con comunidades } \\
\text { cada vez más amplias. }\end{array}$ & 0 & 0 & 0 & 1 & 1 & 1 & 6,25 \\
\hline & TOTAL & 0 & 0 & 0 & 1 & 1 & 1 & 6,25 \\
\hline
\end{tabular}

FUENTE: Elaboración propia.

A continuación proponemos una ejemplificación de los objetivos de las áreas curriculares:

Matemáticas:

- Emplear el ordenador y las TIC para llevar a cabo diferentes cálculos matemáticos y realizar un adecuado tratamiento de la información, así como para aprender y compartir conocimientos (P4e).

Lengua extranjera:

- Utilizar con progresiva autonomía los recursos humanos y materiales a su alcance, incluidas las tecnologías de la información y la comunicación, para obtener información, comunicarse en la lengua extranjera y realizar tareas determinadas ( $\mathrm{P} 4 \mathrm{e})$.

En la dimensión Ideología y valores queda patente la presencia de la competencia en los objetivos de las áreas de Educación artística, Lengua castellana, Lengua extranjera y Conocimiento del medio. No obstante, las áreas de Educación física y Matemáticas quedan sin representación alguna de los aspectos distintivos de la competencia mediática (ver Tabla 5). 
TABLA 5. FRECUENCIAS Y PORCENTAJES TOTALES DE LOS DESCRIPTORES DE LA DIMENSIÓN IDEOLOGÍA Y VALORES

\begin{tabular}{|c|l|c|c|c|c|c|c|c|}
\hline \multicolumn{2}{|c|}{ DESCRIPTORES } & CM & EA & ef & LC & LE & MA & $\%$ \\
\hline \multicolumn{2}{|l|}{$\begin{array}{l}\text { V2a. Habilidad para buscar, organizar, } \\
\text { contrastar, priorizar y sintetizar } \\
\text { informaciones procedentes de distintos } \\
\text { sistemas y de diferentes entornos. }\end{array}$} & 1 & 3 & 0 & 1 & 2 & 0 & 14,58 \\
\hline $\begin{array}{l}\text { V3a. Capacidad de detectar las intenciones } \\
\text { intereses que subyacen tanto en las } \\
\text { producciones corporativas como en } \\
\text { las populares, así como su ideología y } \\
\text { valores, explícitos o latentes, adoptando } \\
\text { una actitud crítica ante ellos. }\end{array}$ & 0 & 1 & 0 & 1 & 0 & 0 & 4,16 \\
\hline TOTAL & 1 & 4 & 0 & 2 & 2 & 0 & 18,75 \\
\hline
\end{tabular}

FUENTE: Elaboración propia.

Los objetivos de las áreas curriculares que proponemos como ejemplo son los siguientes:

1. Conocimiento del medio:

- Identificar, plantearse y resolver interrogantes y problemas relacionados con elementos significativos del entorno, utilizando estrategias de búsqueda y tratamiento de la información, formulación de conjeturas, puesta a prueba de las mismas, exploración de soluciones alternativas y reflexión sobre el propio proceso de aprendizaje (V2a).

2. Educación artística:

- Utilizar la comunicación audiovisual y las TiC para la búsqueda de información [...] (V2a).

3. Lengua castellana:

- Usar y analizar los medios de comunicación social y las tecnologías de la información y la comunicación para obtener, interpretar, valorar e integrar informaciones y opiniones diferentes con un análisis crítico (V3a). 
4. Lengua extranjera:

- Comprender textos audiovisuales (publicidad, películas, informativos) de los medios de comunicación o de las tecnologías de la información y la comunicación, y hacer una lectura crítica y creativa (V3a).

Finalmente, en la dimensión Estética la presencia de la competencia mediática se centra exclusivamente en el área de Educación artística, algo totalmente lógico al tratarse del ámbito de conocimiento propio del área (ver Tabla 6).

\section{TABla 6. FRECUENCIAS Y PORCENTAJES TOTALES DE LOS DESCRIPTORES DE LA DIMENSIÓN ESTÉTICA}

\begin{tabular}{|c|c|c|c|c|c|c|c|c|}
\hline \multicolumn{2}{|r|}{ DESCRIPTORES } & $\mathrm{CM}$ & EA & $\mathrm{EF}$ & LC & LE & MA & $\%$ \\
\hline \multirow{2}{*}{$\begin{array}{l}\frac{n}{2} \\
\frac{1}{2} \\
\frac{1}{4}\end{array}$} & $\begin{array}{l}\text { E1a. Capacidad de extraer placer de los } \\
\text { aspectos formales, es decir, no solo de } \\
\text { lo que se comunica, sino también de la } \\
\text { manera como se comunica. }\end{array}$ & 0 & 1 & 0 & 0 & 0 & 0 & 2,08 \\
\hline & $\begin{array}{l}\text { E2a. Capacidad de relacionar las } \\
\text { producciones mediáticas con otras } \\
\text { manifestaciones artísticas, detectando } \\
\text { influencias mutuas. }\end{array}$ & 0 & 1 & 0 & 0 & 0 & 0 & 2,08 \\
\hline 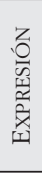 & $\begin{array}{l}\text { E1e. Capacidad de producir mensajes } \\
\text { elementales que sean comprensibles y } \\
\text { que contribuyan a incrementar los niveles } \\
\text { personales o colectivos de creatividad, } \\
\text { originalidad y sensibilidad. }\end{array}$ & 0 & 1 & 0 & 0 & 0 & 0 & 2,08 \\
\hline & TOTAL & 0 & 3 & 0 & 0 & 0 & 0 & 6,25 \\
\hline
\end{tabular}

FUENTE: Elaboración propia.

Los ejemplos más significativos quedan expresados a continuación:

Educación artística:

- Desarrollar la capacidad de observación y la sensibilidad para apreciar las cualidades estéticas, visuales y sonoras del entorno (E1a).

- Desarrollar confianza en las elaboraciones artísticas propias, disfrutando con su realización y apreciando su contribución al crecimiento personal $y$ del grupo, para afianzar su autoestima y mejorar la capacidad de expresión de ideas y sentimientos (E1e). 


\section{DisCUSIÓN DE LOS RESUlTADOS Y CONCLUSIONES}

La trascendencia de los medios de comunicación en nuestras vidas es de tal magnitud que autores como Jenkins (2008; 2009, 29) han analizado en profundidad las implicaciones sociales y educativas de lo que se ha considerado el nuevo entorno comunicativo. Para este autor es necesario indagar tanto sobre las "new media literacies" como sobre las tradicionales, ya que ambas inciden de manera directa sobre el ciudadano e incrementan su confusión entre la representación y la realidad.

La necesidad de acometer una verdadera educación en medios es algo inaplazable, autores como Scheuer $(2009,15)$ manifiesta que "desde hace más de veinte años, educadores de todo el mundo han hecho campaña a favor de la educación en medios; sin embargo, en la mayoría de los países, los responsables de las políticas educativas nacionales no han advertido hasta muy recientemente la importancia de la alfabetización mediática".

Nuestro sistema educativo, alojado dentro de la órbita europea, ha iniciado recientemente una reforma que, junto a la calidad y la equidad de la educación, encuentra en las competencias básicas el eje rector de la nueva configuración del sistema educativo y del currículo escolar (Tiana, 2011). Si bien la competencia mediática no constituye una de las ocho competencias básicas, esta aparece explicitada tanto en los objetivos de la etapa de educación primaria, como en los objetivos de las áreas que conforman el currículo de la misma.

En el análisis realizado a los objetivos de área esta presencia se ha cifrado en un $36,36 \%$, lo que supone un porcentaje importante respecto a la incertidumbre inicial que planteábamos.

En este análisis y de acuerdo con lo manifestado por Tucho (2008), podemos destacar dos enfoques: por un lado, la consideración de los medios de comunicación como instrumentos de apoyo a los procesos de enseñanza y aprendizaje; por otro lado, la necesidad de un análisis de estos desde una perspectiva crítica y desde su repercusión social.

Este primer enfoque es el que ha contado con una mayor generalización en el currículo escolar, mientras que el segundo ha quedado a la buena voluntad del profesorado, sobre todo a partir de la incorporación de las tecnologías de la información y la comunicación a dicho currículo. No obstante, esta aparición de las TIC en los centros educativos ha originado una mayor acción sobre descriptores de la competencia mediática que de otro modo hubiese sido inimaginable.

Asimismo, entendemos que el estudio presentado da un paso más en esta visión polarizada de la presencia de los medios de comunicación en la normativa estatal y autonómica. Al partir de una delimitación de la competencia mediática en seis dimensiones y dos ámbitos de desarrollo -análisis y expresión-, posibilita un análisis pormenorizado de la misma en los objetivos de área, destacando que: 
1. Si bien no todos los descriptores de la competencia mediática quedan vinculados con los objetivos de área todas las dimensiones quedan reflejadas en dichos objetivos.

2. En todas las áreas se aprecia alguna relación entre los descriptores de la competencia mediática y sus objetivos.

3. La dimensión Lenguajes es la que mayor presencia obtiene en los objetivos de área con un 33,33\%, seguida de Tecnología e Ideología y valores con un $18,75 \%$ cada una, Procesos de interacción con un $16,66 \%$ y finalmente Procesos de producción y difusión y Estética con un 6,25\% cada una de ellas. Esta distribución puede ser perfectamente entendible, ya que es el lenguaje el que da forma a dos de las áreas del currículo consideradas como instrumentales -Lengua castellana y Lengua extranjera-. En segundo lugar, la incorporación de la competencia digital al currículo ha podido provocar la presencia en el mismo de la dimensión Tecnología y Procesos de interacción. Así como el hincapié que se ha hecho en la explicitación curricular de la educación en valores, los tradicionalmente llamados temas transversales. En tercer lugar, la edad del alumnado puede condicionar una mayor ausencia de la dimensión Procesos de producción y difusión debido a que esta requiere una mayor madurez en el alumnado. Finalmente, la Estética ha constituido un aspecto tratado casi en exclusiva desde el área de Educación artística y esta se distribuye en dos materias, Educación plástica y Música, lo que también ocasiona su propia presencia tangencial en el currículo escolar.

4. Las dimensiones Lenguajes y Tecnología son las únicas que manifiestan su presencia en todas las áreas curriculares de la etapa de educación primaria.

5. Todas las dimensiones de la competencia mediática, salvo Estética, se encuentran presentes en las áreas de Lengua castellana y Lengua extranjera.

En definitiva, aunque la competencia mediática aparece reflejada en los objetivos de las áreas del currículo de la etapa de educación primaria, es necesario que esta se explicite aun más, de manera que el docente en su proceso de concreción curricular la identifique y seleccione aquellos objetivos que posibiliten realmente poner en marcha programas de alfabetización mediática en las aulas.

\section{REFERENCIAS BIBLIOGRÁFICAS}

Aguaded, J. I. et al. (invs.) (2011) El grado de competencia mediática en la ciudadanía andaluza. Huelva, Grupo Comunicar Ediciones/Grupo de Investigación Ágora, Universidad de Huelva.

Aparici, R.; Campuzano, A.; Ferrés, J. y García, A. (2010) La educación mediática en la escuela 2.0. Consultado el 23 de mayo de 2011.

http://ntic.educacion.es/w3/web_20/informes/educacion_mediatica_e20_julio20010.pdf. 
ARnaiz, P. y De Haro, R. (2004) Ciudadanía e interculturalidad: claves para la educación del siglo XXI. Educatio, 22, 19-37.

Arroyo, J. A. (2009). Gestión directiva del currículum. Actualidades Investigativas en Educación, 9 (2), 1-17. http://redalyc.uaemex.mx/src/inicio/ArtPdfRed.jsp?iCve= 44713058021.

Ausubel, D. P. (1976) Psicología Educativa. Una perspectiva cognitiva. México, Trillas.

Bloom, B. S. (1956) Taxonomy of educational objectives; the classification of educational goals. New York, Longmans, Green.

Braslawsky, C. (2001) Tendances mondiales et développement des curricula, communication au Colloque international, L'éducation dans tous ses états: influences européennes et internationales sur les politiques d'éducation et de formation. Bruxelles: Association Francophone d'Éducation Comparée.

CAmps, V. (2009) La educación en medios, más allá de la escuela. Comunicar, 32, 139-145. http://dx.doi.org/10.3916/c32-2009-02-012.

Coll, C. y MarTín, E. (2006) Vigencia del debate curricular. Aprendizaje básicos, competencias y estándares. II Reunión del Comité Intergubernamental del Proyecto Regional de Educación para América Latina y el Caribe (PRELAC). Santiago de Chile, 11-13 de mayo de 2006. Documento no publicado. Consultado el 15 de febrero de 2013. http://www.ub.edu/grintie.

De Ketele, J. M. (2008) Enfoque socio-histórico de las competencias en la enseñanza. Profesorado. Revista de Currículum y Formación del Profesorado, 12 (3), 1-12.

De la ORden, A. (2011) El problema de las competencias en la educación general. Bordón. Revista de Pedagogía, 63 (1), 47-61.

EsCAMILla, A. (2009) Las competencias en la programación de aula. Infantil y primaria (3-12 años). Barcelona, Graó.

FERRÉs, J. (2007) La competencia en comunicación audiovisual: propuesta articulada de dimensiones e indicadores. Comunicar, 29, 100-107.

FERRÉs, J. et al. (2011) Competencia mediática. Investigación sobre el grado de competencia de la ciudadanía en España. Madrid, Ministerio de Educación.

FERRÉs, J. y PISCITElLI, A. (2012) La competencia en educación mediática: dimensiones e indicadores. Comunicar, 38, 75-82.

GonzÁlez Fernández, N.; SedeÑo, M. y GonZÁlvez, V. (2012) Diseño de un focus group para valorar la competencia mediática en escenarios familiares. Icono, 14, 10 (3), 116-133.

GOÑI, J. M. (2009) El desarrollo de la competencia matemática en el currículo escolar de la Educación Básica. Educatio Siglo XXI, 27 (1), 33-58.

Jenkins, H. (2008) Convergence Culture. Una cultura de la convergencia. Barcelona, Paidós.

Jenkins, H. et al. (2009) Confronting the Challenges of Partipatory Culture. Media Education for the 21st Century. Massachusetts, The MIT Press.

Johnson, H. (1982) Currículum y Educación. Barcelona, Paidós.

Jonnaert, P.; Barrette, J.; Masciotra, D. y Yaya, M. (2008) La competencia como organizadora de los programas de formación: hacia un desempeño competente. Profesorado. Revista de Currículum y Formación del Profesorado, 12 (3), 1-32. Consultado el 12 de enero de 2012.

http://www.ugr.es/local/recfpro/rev123ART3.pdf.

Le Boterf, G. (2000) Ingeniería de las competencias. Barcelona, Gestión 2000/EPISE.

Ley Orgánica 2/2006, de 3 de mayo, de Educación. BOE n.ํㅜ 106, de 4 de mayo de 2006. 
Medrano, M. C. (2008) Televisión y educación: del entretenimiento al aprendizaje. Teoría de la Educación. Revista Interuniversitaria, 20, 205-224.

MinISTERIO DE EDUCACIÓN (2004) Una educación de calidad para todos y entre todos. Papeles para el debate. Madrid, MEC.

OCDE-DESECO (2002) Definition and Selection of Competencies: Theoretical and Conceptual Foundations. Consultado el 17 de febrero de 2011. http://www.portal-stat.admin.ch/deseco/index.htm.

Perrenoud, P. (2004) Diez nuevas competencias para enseñar. Barcelona, Graó.

REAL DECRETO 1513/06, de 7 de diciembre, por el que se establecen las enseñanzas mínimas de educación primaria. Boletín Oficial del Estado número 293, de 8 de diciembre de 2006.

Scheuer, M. (2009) Prólogo. Comunicar, XVI, 32, pp. 15-16. http://dx.doi.org/10.3916/c32-2009-01-002.

TABA, H. (1974) Elaboración del Currículo. Buenos Aires, Troquel.

TARDIF, J. (2008) Desarrollo de un programa por competencias: De la intención a su implementación. Profesorado. Revista de Currículum y Formación del Profesorado, 12 (3), 1-16. Consultado el 12 de enero de 2012. http://www.ugr.es/local/recfpro/rev123ART2.pdf.

Tiana, A. (2011) Análisis de las competencias básicas como núcleo curricular en la educación obligatoria española. Bordón. Revista de Pedagogía, 63 (1), 63-75.

ToBón, T. S. (2006) Formación basada en competencias. Pensamiento complejo, diseño curricular y didáctica. Bogotá, ECOE Ediciones.

Tucho, F. (2008) La educación en comunicación en la LOE y sus decretos de Enseñanzas Mínimas. Comunicar, 31, 547-553.

ZABALA, A. y ARNAU, L. (2008). 11 ideas clave. Cómo aprender y enseñar competencias. Barcelona, Graó. 